\title{
A Numerical Model for Computation the Optimum Value of Second Gruneisen Parameter (q) for Copper
}

\author{
Dr. Janan F. Ahmad \& Dr. Mumtaz M. Saleh \\ Department of Physics / College of Education \\ University of Mosul \\ \& \\ Dr. Adnan M. Al-Sheihk \\ Department of Physics / College of Science \\ University of Mosul
}

Received

14 / 09 / 2009
Accepted

$06 / 10 / 2010$

\section{ملخص البحث}

في هذا البحث تم صياغة نموذج امتلية عددية يعتمد على القيم التجريبية لكل من

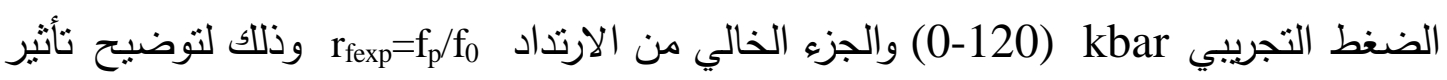

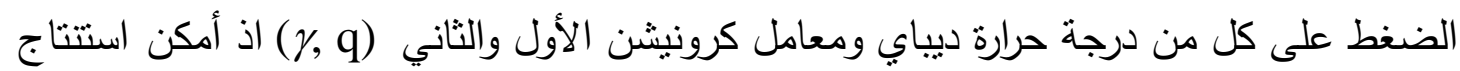

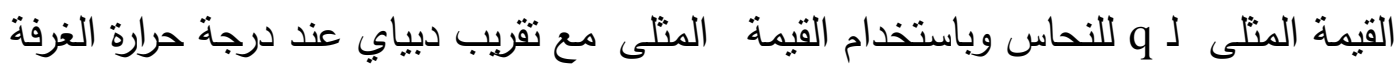

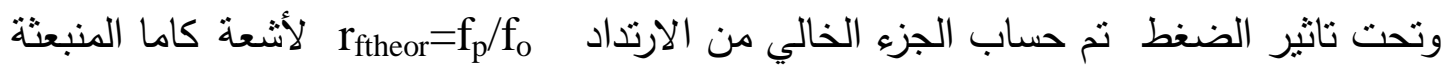
من

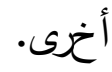

\begin{abstract}
In the present work a numerical optimization model is constructed which is based on the experimental pressure $\left(\mathrm{p}_{\exp }\right)$ and experimental recoilless fraction ratio $\left(\mathrm{r}_{\text {fexp }}=\mathrm{f}_{\mathrm{p}} / \mathrm{f}_{0}\right)$ data in order to explain the pressure effect on Gruneisen parameters $(\gamma, q)$ and Debye temperature. The model was able to compute the optimum q value for copper, the observed optimum value for $\mathrm{q}$ was used with Debye approximation at room temperature under pressure to calculate the recoilless fraction ratio $\mathrm{r}_{\mathrm{ftheor}}=\mathrm{f}_{\mathrm{p}} / \mathrm{f}_{\mathrm{o}}$ for $\left(\mathrm{Fe}^{57}-\mathrm{Cu}\right)$, It has been found that the calculated results of (ffraction) are in good agreement with the experimental data of recoilless fraction ratio.
\end{abstract}




\section{1- INTRODUCTION:}

The Gruneisen parameter $\gamma$ is an important physical quantity in solving many research problems of condensed matter physics and geophysics. The study of pressure (volume) dependence of $\gamma$ is interesting problem from theoretical as well as experimental point of view, particularly, due to the lack of a proper theory and enough experimental data (Fang 1996).

In the reduction of shock-wave data to isothermal data, the knowledge of pressure dependence of the Gruneisen parameter is very useful. The volume variation of $\gamma$ is central in theoretical equation of state, geophysical models, ultrasonic measurements and melting of solids (Pandya et al 2002). The Gruneisen parameter $\gamma$ varies slowly as a function of pressure, it has both a microscopic and macroscopic definitions (Vocadlo L., etal 2003), the former rlating it to the virational frequencies of atoms in amaterial in the following form

$\gamma=-\partial \ln \omega_{\mathrm{i}} / \partial \ln v$

Where

$\mathrm{v}$ - volume

$\omega_{i}$ - frequency of the ith mode of vibration of the lattice

The experimental values of Gruneisen parameters are mostly available at normal pressure (Gschneidner 1964). The volume variation of $\gamma$ can be obtained from experimental measurements of change of temperature with small adiabatic pressure changes (Ramakrishnan et al 1978). In such an experiment a substance is generally compressed by about $10 \%$ and for higher compressions, $\gamma$ values are extrapolated by using the relation

$\gamma=\gamma_{0}\left(\mathrm{~V}_{\mathrm{p}} / \mathrm{V}_{0}\right)^{\mathrm{q}}$

where $\mathrm{q}$ is a Gruneisen parameter. $\mathrm{V}_{0}$ and $\mathrm{V}_{\mathrm{p}}$ are volumes at zero pressure and the pressure under consideration, respectively. Several expressions have been proposed in the literature to calculate this parameter (Grover et al 1969; Godwal et al 1983; Kumari and Das 1986).

The volume dependence of $\gamma$ is given by the relation $\mathrm{q}=\mathrm{d} \ln \gamma / \mathrm{d} \ln \mathrm{V}$

the value of $q$ is generally not equal to unity. Recently, (Nie 2000) has suggested the following form for the volume dependence of $\gamma$ $\gamma=\gamma_{\mathrm{o}} \exp \left[\mathrm{q}_{0} / \mathrm{n}\left(\mathrm{v}_{\mathrm{p}} / \mathrm{v}_{\mathrm{o}}\right)^{\mathrm{n}}-1\right]$

Where:

$\gamma$-Gruneisen parameter at pressure P.

$\gamma_{0^{-}}$1st Gruneisen parameter at zero pressure (atmospheric).

$\mathrm{v}_{\mathrm{p}}$ - specific volume at pressure $\mathrm{p}$

$\mathrm{v}_{\mathrm{o}}$ - specific volume at zero pressure

$\mathrm{n}$ - value depend on matter 
Attempts have also been made to compute $\gamma$ and its volume dependence using model approaches. Moriarty et al (1984) and Soma et al (1983) have computed the volume variation of $\gamma$ for $\mathrm{Al}$ and alkali metals respectively using the pseudopotential approach (Godwal et al 1979, 1983). Nagara and Nakamura (1984) have been used the ThomasFermi-Dirac statistical model to compute $\gamma$ for $\mathrm{Al}$ and $\mathrm{Fe}$ as function of volume. Bratkovskii et al (1984) have also carried out similar studies for several metals. We are in the present paper, construct a numerical model to compute the volume variation of the Grunisen parameters to get the optimum value of $\mathrm{q}$ for Copper by using Debye approximation for the pressure effect on the recoilless fraction for $\mathrm{Fe}^{57}: \mathrm{Cu}$.

\section{2- Theoretical details}

\subsection{The f-fraction (recoilless fraction)}

The recoilless fraction are those nuclear transitions in which the lattice is in the same quantum state after the transition as before the transition i.e no phonon is emitted, thus the f-value for a certain initial lattice state $\left\{\mathrm{n}_{\mathrm{s}}\right\}$

$\mathrm{f}\left\{\mathrm{n}_{\mathrm{s}}\right\}=\left|<\left\{\mathrm{n}_{\mathrm{s}}\right\}_{\mathrm{g}}\right| \mathrm{H}\left|\left\{\mathrm{n}_{\mathrm{s}}\right\}_{\mathrm{e}}>\right|^{2}$

Where:

$\left\{\mathrm{n}_{\mathrm{s}}\right\}=\mathrm{n}_{\mathrm{s}}$ phonon in the Sth lattice mode.

The subscripts $g$ and e denote the ground and excited states of the nucleus, respectively and $\mathrm{H}$ represents non relativistic Hamiltonian responsible for this decay. By taking the ratio of those transitions in which the lattice remains in the same state of the (zero phonon transition) to the total transitions, the nuclear matrix elements cancel and one get

$\mathrm{f}\left\{\mathrm{n}_{\mathrm{s}}\right\}=\left|<\left\{\mathrm{n}_{\mathrm{s}}\right\}_{\mathrm{g}}\right| \mathrm{e}^{\text {ik. }} \chi\left|\left\{\mathrm{n}_{\mathrm{s}}\right\}_{\mathrm{e}}>\right|^{2}$

Where:

$\mathrm{k}-$ wave vector of the emitted radiation

$\chi$ - center of mass of decaying nucleus

In Debye approximation, f-fraction of dilute alloy of $\mathrm{Fe}^{57}$ in $\mathrm{Cu}$ at $P$ pressure expressed as (Moyzis 1968)

$f_{p}=\exp \left[-6 E_{R} T / k_{B} \theta_{p}{ }^{2}\right]$
$f_{0}=\exp \left[-6 E_{R} T / k_{B} \theta_{D}{ }^{2}\right]$
$r_{\text {ftheor }}=f_{p} / f_{o}$

$\mathrm{T} \geq \theta_{\mathrm{D}}$

Where:

$f_{p}-f$-fraction at pressure $p$-kbar.

$\mathrm{f}_{\mathrm{o}}$ - $\mathrm{f}$-fraction at atmospheric pressure (zero kbar).

$\mathrm{E}_{\mathrm{R}}-$ recoil energy of free nucleus due to decay $=\mathrm{E}_{\gamma}{ }^{2} / 2 \mathrm{MC}^{2}$

$\mathrm{M}$ - Nuclear mass

$\mathrm{E}_{\gamma}$ - Gamma-ray energy

C- speed of light 
$\mathrm{T}-300 \mathrm{~K}$

$\mathrm{k}_{\mathrm{B}}$ - Boltzman constant

$\theta_{\mathrm{D}}-$ Debye temperature at atmospheric pressure (tight lattice binding) $=314 \mathrm{~K}$

$\theta_{\mathrm{p}}-$ Debye temperature at pressure $\mathrm{P}$ kbar

\subsection{Pressure dependence of Debye temperature:}

The Variation of Debye temperature due to pressure effect is (Dubrovinsky et al 2000) expressed as:

$\left.\theta_{P}=\theta_{D} \exp \left(\gamma_{0} / \mathrm{q}\left(1-\mathrm{V}_{\mathrm{p}} / \mathrm{V}_{0}\right)^{\mathrm{q}}\right)\right)$

$\theta_{\mathrm{p}}$ - can be calculated as in Eq.(8) while $\mathrm{V}_{\mathrm{p}} / \mathrm{V}_{0}$ can be evaluated by Murnagphan equation of state.

$\mathrm{V}_{\mathrm{p}} / \mathrm{V}_{0}=\left(1+B_{s}^{-} \mathrm{P} / \mathrm{B}_{\mathrm{OS}}\right)^{1 / B_{s}^{-}}$

$\mathrm{P}$ - pressure in kbar

$\mathrm{B}_{\mathrm{OS}}-$ Adiabatic bulk modulus at atmospheric pressure of copper $=1331.5$ kbar.

$B_{s}^{-}=$pressure derivative of adiabatic bulk modulus at atmospheric pressure $=5.68$ (Moyzis 1968).

\section{3- Computation and results:-}

We are used a computer program for the volume and pressure effect on Grunisen parameters $(\gamma, q)$ as shown in fig. (1) which describe the steps of program to calculate the optimum value of $\mathrm{q}$ for Copper which is equal (1.03) as shown in fig.(2) and by using this value of q, the first Grunisen parameter $\gamma_{0}$ has been calculated which equal (1.96).

The variation of first Grunisen parameter $\gamma$ with compressed volume has been obtained by using equ.(2), fig.(3) shows the first Grunisen parameter $\gamma$ decreases slowly with compressed volume and this result is the same as the one obtained by (Pandya et al 2002) who take the compression volume up to $40 \%$ by using another formula, table (1) show the comparation of $\left(\gamma_{0}, q\right)$ with experimental and theoretical values for copper. Number of authors, in their high pressure studies have used the approximation $\gamma(\mathrm{V})=$ constant (Altshuler etal 1987; Ob etal 1991) Murnaghan equation (9) which has been used for low compression up to $10 \%$ i.e. $\mathrm{V}_{\mathrm{p}} / \mathrm{V}_{\mathrm{o}} \geq 0.9$ as shown in fig (3).

By using the value of $\gamma_{0}, \mathrm{q}$, the pressure effects on Debye temperature has been calculated by equation (8) (Dubrovinsky etal 2002).

From the value of Debye temperature and Debye approximation we calculate the pressure effects on the recoilless fraction ratio $r_{\text {ftheor }}=f_{p} / f_{o}$ for $\left(\mathrm{Fe}^{57}-\mathrm{Cu}\right)$ and compare with experimental recoilless fraction ratio $\left(\mathrm{r}_{\text {fexp }}=\mathrm{f}_{\mathrm{p}} / \mathrm{f}_{0}\right)$ (Moyzis 1968) as shown in fig. (4) we get good fitting.

Gruneisen parameter $\gamma$ decrease slowely with applied pressure for $\mathrm{Cu}$ which is one of Fcc transition metals and this result agree with the other auther result (Pandya et al 2002). 


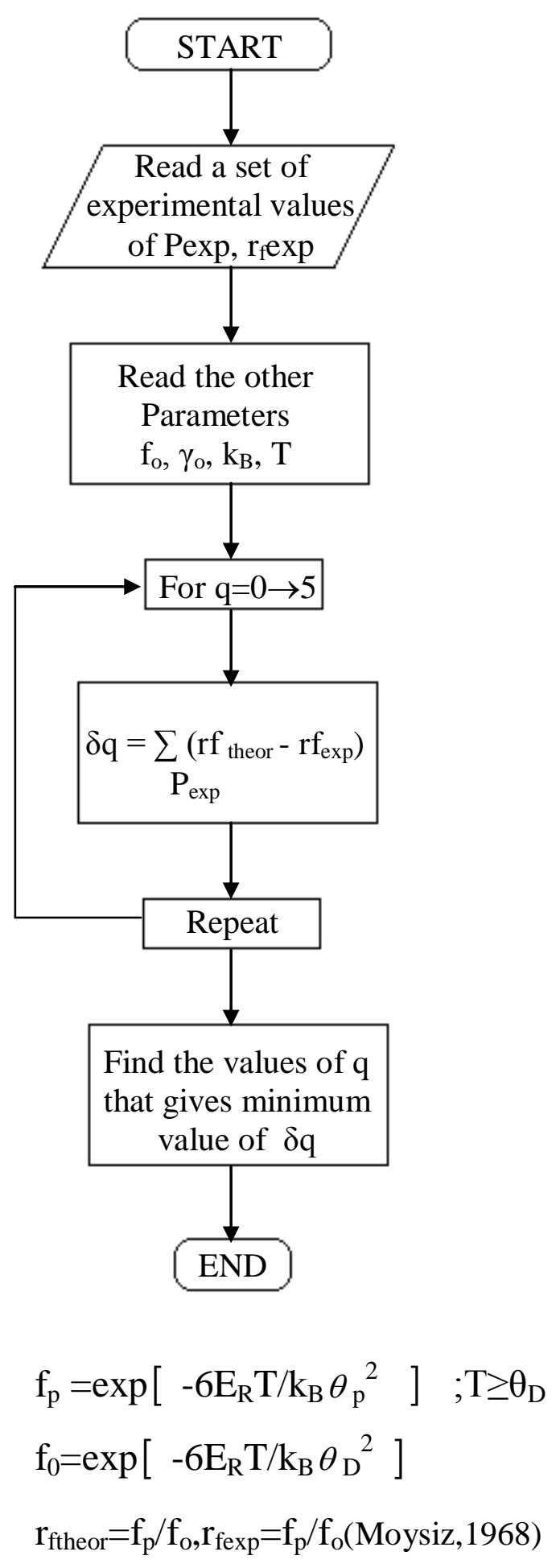

Fig.(1): Flow chart to calculate the optimum value q for Copper 


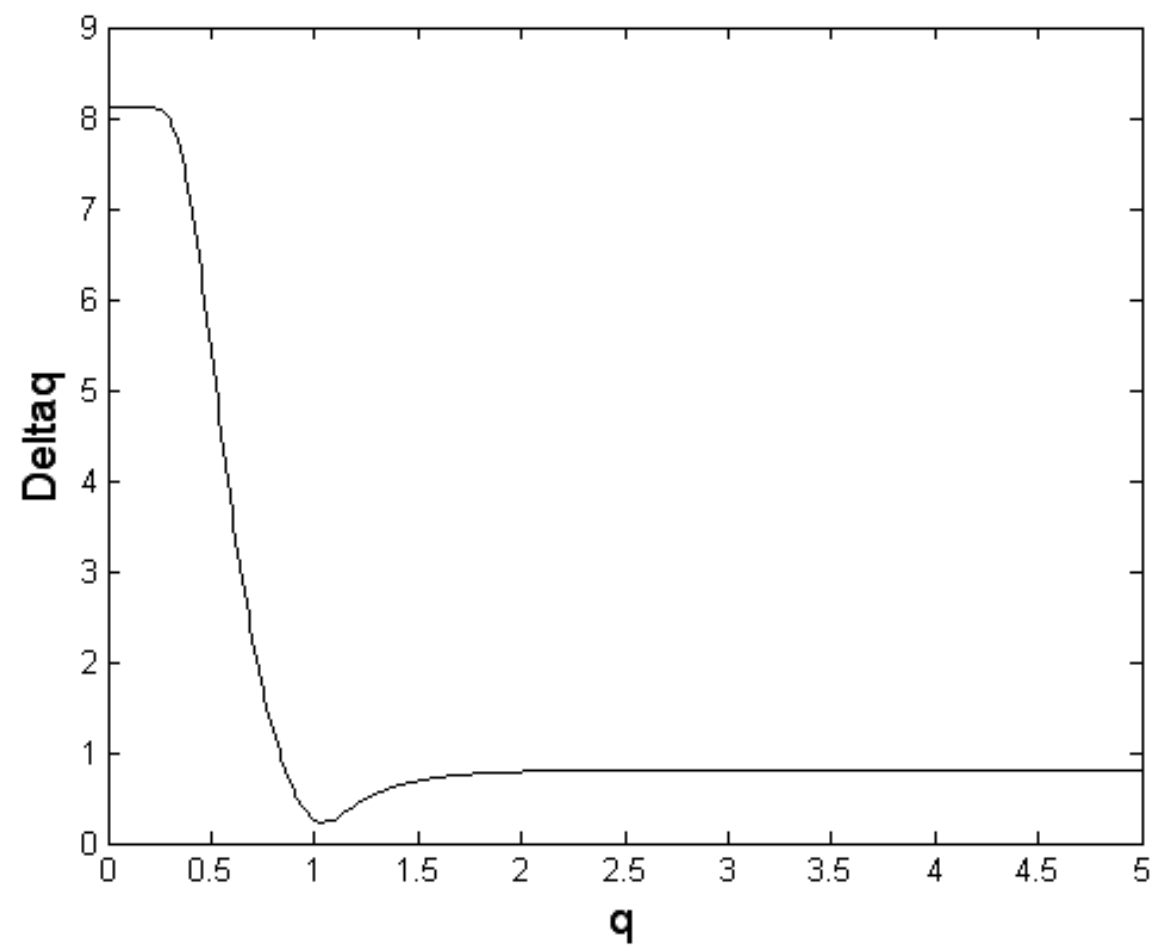

Fig (2): optimum value $q$ for $\mathrm{Cu}$

Table -1: Comparation of experimental and theoretical values $\left(\gamma_{0}, q\right)$ for copper in pressure range $(0-100) \mathrm{kbar}$

\begin{tabular}{|c|c|c|c|}
\hline \multirow{2}{*}{$\begin{array}{c}\text { Experimental results } \\
\qquad \gamma_{0}\end{array}$} & \multicolumn{3}{|c|}{ Theoretical results } \\
\hline & $\mathbf{q}$ & $\gamma_{0}$ & \\
\hline $\begin{array}{c}1.96,2 \pm 0.06 \\
\text { (Gschneidner 1964) }\end{array}$ & 1.03 & 1.96 & (Present work) \\
\hline \multirow{8}{*}{$\begin{array}{c}1.97,1.96,2 \pm 0.08 \\
\text { (Gschneidner 1964) }\end{array}$} & 1.08 & 1.93 & (C V Pandya etal,2002) \\
\hline & 1.33 & 2.01 & (Ramakrishnan etal, 1978) \\
\hline & & 1.84 & (Barrera and Batana 1993b) \\
\hline & & 1.73 & (Daniels and Smith 1958) \\
\hline & & 1.19 & (Harrison and Wills 1983) \\
\hline & & 1.9 & (Pal and Sengupta 1979) \\
\hline & & 2.15 & (Pal and Sengupta 1979) \\
\hline & & 2. 16 & (Pal and Sengupta 1979) \\
\hline
\end{tabular}




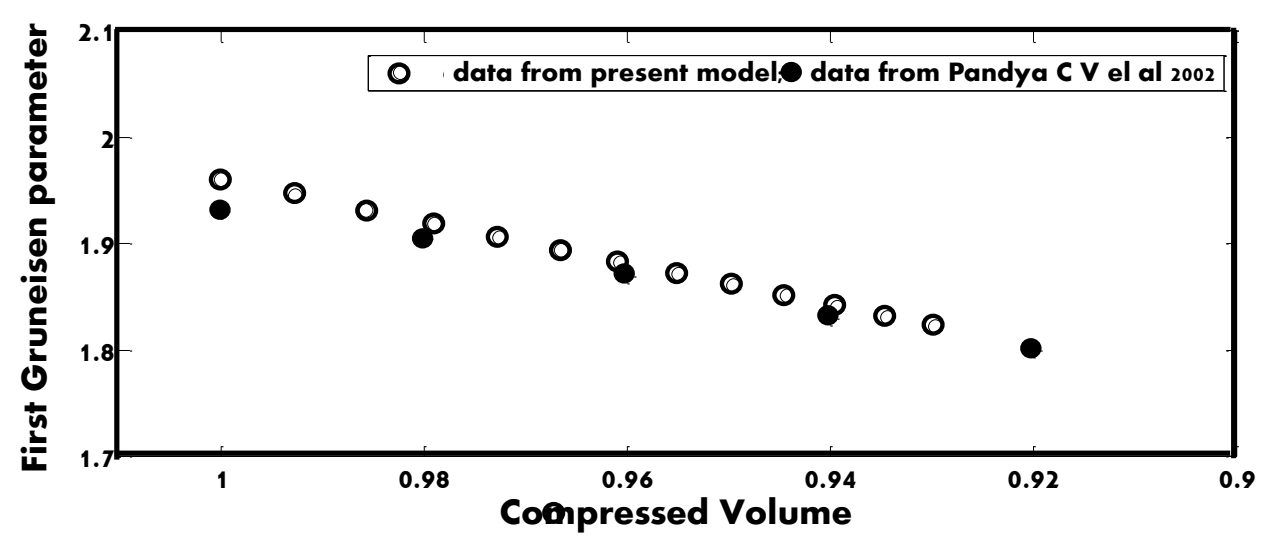

Fig(3): The volume variation of first Gruneisen parameter $\gamma$

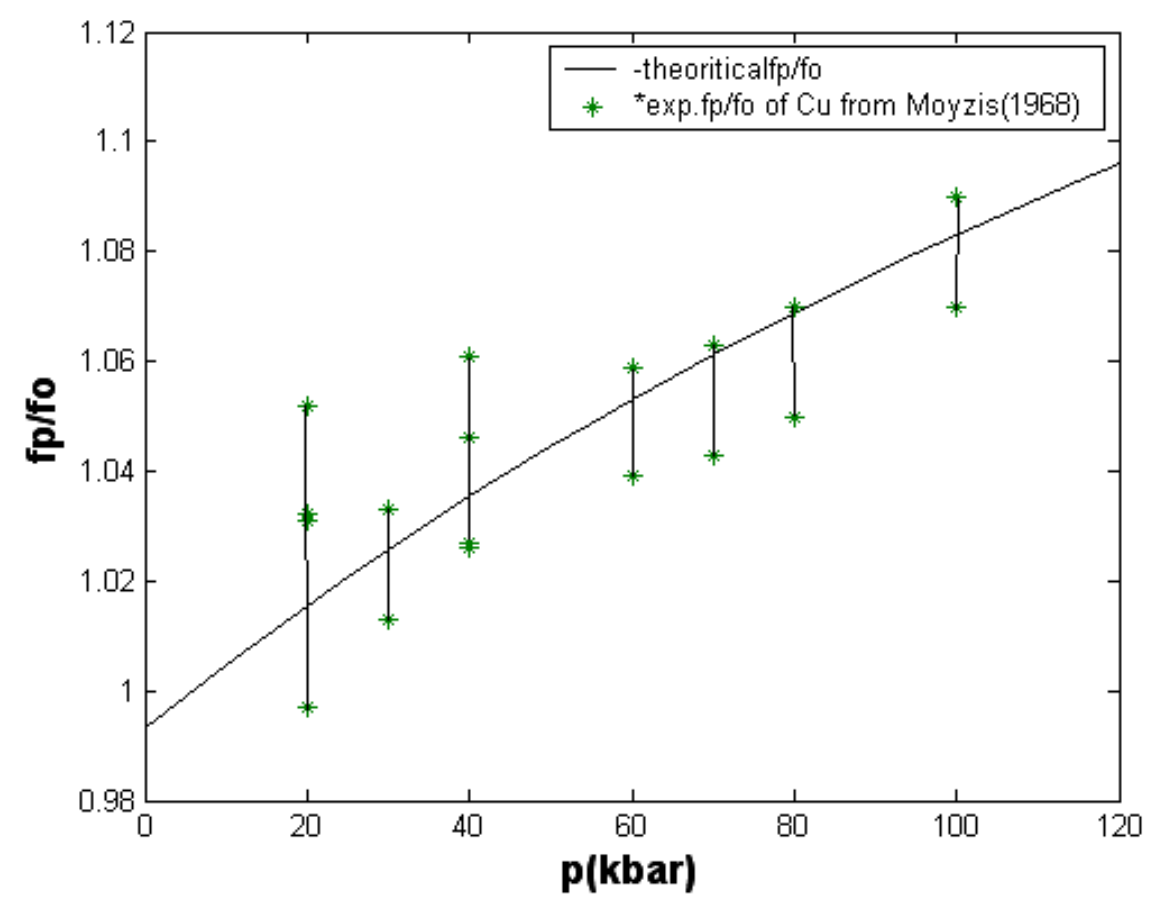

Fig (4): Fitting between the theortical and expermental values of recoilless fraction ratio $\left(\mathbf{f}_{\mathrm{p}} / \mathbf{f}_{\mathbf{o}}\right)$ for $\left(\mathrm{Fe}^{57}-\mathrm{Cu}\right)$

\section{4- Conclusions}

We use one of the application of the Mossbauer effect (experimental recoilless fraction ratio $\left(\mathrm{r}_{\text {fexp }}=\mathrm{f}_{\mathrm{p}} / \mathrm{f}_{0}\right)$ data) to study the pressure effect on Gruneisen parameters $(\gamma, q)$ by proposing a numerical program to find the optimum value of $\mathrm{q}$.

The obtained value of $\mathrm{q}$ give a good fiting between the theoretical and experimental recoilless fraction ratio $\mathrm{f}_{\mathrm{p}} / \mathrm{f}_{0}$. The theoretical recoilless fraction ratio $\mathrm{f}_{\mathrm{p}} / \mathrm{f}_{0}$ increased slowly with the applied pressure.

Debye temperature $\theta_{P}$ increased linearly with applied pressure from $\theta_{D}=314 \mathrm{~K}$ to $\theta_{P}=358.68 \mathrm{~K}$ when the pressure was increased from (0-120)Kbar. 


\section{References}

1) Altshuler L. V., Brusnikin S. E. and Kazmenkov E. A. 1987 J. Appl. Mech. Tech. phys. 28129.

2) Barrera G D and Batana A 1993b Phys. Rev. B47 8588

3) Bratkovskii A M, Vaks V G and Trefilov A V 1984 Sov. Phys. JETP 591245.

4) Daniels W B and Smith C S 1958 Phys. Rev. 111713.

5) Dubrovinsky L S, Saxena S K, Dubrovinskaia N A, Rekhi S, and Le Bihan T, 2000,V.85, 386-389.

6) Fang Z H 1996 J. Phys. Condens Matter 87067.

7) Fang Z H and Rong L 1994 J. Phys. Condens Matter 66937 Godwal B K, Sikka S K and Chidambaram R 1983 Phys. Rep. 102 121.

8) Grover R, Keeler R N, Rogers F J and Kennedy G C 1969 J. Phys. Chem. Solids 302091.

9) Gschneidner K A 1964 Solid state phys. advances in research and applications (eds) F Seitz and D Turnball (New York: Academic Press) 16 p. 412.

10) Harrison W A and Wills J M 1983 Phys. Rev. B28 4363.

11) Kumari M and Das N 1986 Phys. Status Solidi (b)133 101.

12) Leibfried G and Ludwig W 1969 Solid state physics (eds) F Seitz and D Turnball (New York: Academic Press) 12.

13) Moriarty J A, Toung D A and Ross M 1984 Phys. Rev. B30 578.

14) Moyzis, J.A., (1968), "High pressure Mossbauer studies on iron and its dilute alloys", Ph.D. thesis, Univ. of Illinois, USA.

15) Nagara $\mathrm{H}$ and Nakamura $\mathrm{T} 1984$ Phys. Rev. B31 1844.

16) Nie C H 2000 Phys. Status Solidi (b)219 241.

17) Ob K. H. Jhung K. G., Kim I. H. and Persson P. A. 1991 Shock compression of condensed matter, Proc. Amer. Phys. Soc. Topical conference p.103.

18) Pal S K and Sengupta S P 1979 Indian J. Phys. A53 225.

19) PANDYA $C$ V, VYAS $P$ R, ANDYA $T C P$ and GOHEL $V B$ Bull. Mater. Sci., Vol. 25, No. 1, February 2002, pp. 63-67. (C) Indian Academy of Sciences.

20) Parshukov A V 1985 Sov. Phys. Solid State 27741.

21) Parshukov A V and Batsanov S S 1984 Sov. Phys. Solid State 26 2064 Wallace D C 1992 Phys. Rev. B46 5242.

22) Ramakrishnan J, Boehle R, Higgins G H and Kennedym G C 1978 J. Geophys. Res. 833335.

23) Soma T, Kagaya H M and Nishigaki M 1983 Phys. Status Solidi (b)116 673 .

24) Vocadlo L., Alfe D., Gillan M. J. and Price G.D., 2003, The properties of iron under core condition from first principles calculations phys. Of the earth and planetary interiors,140,101-125. 